\title{
Thermal Wave Detection and Ranging for Non-destructive Testing and Evaluation
}

\author{
Vanita Arora and Geetika Dua \\ InfraRed Imaging Laboratory (IRIL), Department of Electrical Engineering, Indian Institute of \\ Technology Ropar, Nangal Road, Rupnagar, Punjab, India-140001
}

Tel: +91-1881-222118 fax: +91-1881-227078

*ravibabucareiitd@yahoo.co.in

\begin{abstract}
Thermal Wave Detection and Ranging (TWDAR) plays a vital role for non-destructive testing and evaluation (NDT\&E) of various solid materials due to it's whole field, remote and quantitative inspection capabilities to reveal the surface or subsurface anomalies. This can be realized, by recording the temperature distribution over the test sample for the predefined incident thermal excitation. Present work proposes recent trends in non-stationary thermal imaging modalities and the associated pulse compression favourable post processing approaches which can be releasable experimentally by using low peak power heat sources than the widely used conventional pulsed thermographic methods (PT \& PPT) and in very less time compared to sinusoidal modulated Lock-in Thermography (LT). Furthermore, results obtained with various non-stationary thermal imaging techniques are compared with the conventional frequency domain phase based post processing technique.
\end{abstract}

Keywords: Frequency modulation, non-stationary signals, non-destructive testing, matched filters, phase images. 